\title{
Artifact-Free, Long-Lasting Phase Plate
}

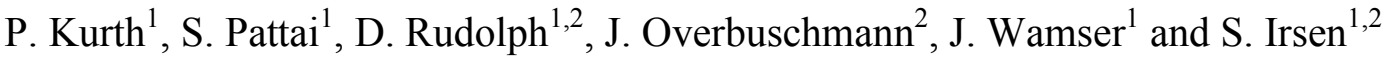 \\ 1. KonTEM GmbH, Bonn, Germany \\ 2. Center of Advanced European Studies and Research (caesar), Electron Microscopy and Analysis \\ (EMA), Bonn, Germany
}

With recent advances in sample preparation and TEM instrumentation, cryo transmission electron microscopy (cryo-TEM) has become an important and powerful technique in structural biology to analyze specimen in their native state [1]. These frozen, hydrated specimen however show only weak image contrast. Nearly all image information is contained in a phase change of the scattered electron beam. This phase information can be transferred to image contrast by influencing the contrast transfer function (CTF) of the TEM. This is commonly done by changing the focus ("defocusing"). Unfortunately, at the same time, image resolution is lowered. In addition, cryo-samples are too beamsensitive to record multiple images of the same spot to compensate for the worsened resolution caused by the defocusing.

Using a phase plate instead of defocusing is a viable alternative to gain contrast and preserve resolution. The phase plate is inserted into the back focal plane of the TEM at or near Gaussian focus, modulating the CTF. The phase of the scattered electrons is shifted by $\pi / 2$. This allows maximum contrast transfer. Thus, phase plates can show structures that are not visible in the original data gained by defocusing. This holds particular advantage for objects which cannot be averaged [2].

So far, the main obstacles for the widespread use of phase plates were their limited lifetime and the prevalence of image artifacts. Here, we present procedures and techniques that solve the above mentioned problems.

As an alternative to prevalent carbon-film and multi-layer Zernike type phase plates, we developed a new, self-supporting, 1-layer thin film metal phase plate. The phase shift of $\pi / 2$ is controlled by the thickness of the layer with respect to the desired accelerating voltage. To let pass the unscattered electron beam, a central hole is milled into the film using a focused ion beam system (FIB).

Furthermore, we devised an in-situ conditioning process that removes any remaining hydrocarbons from the phase plate. After this treatment, the phase plate shows no signs of ageing.

In addition, we implemented the rocking beam technique to remove eventual charging of the phase plate aperture [3] and to realize a time-averaged image. Alternatively, we let rotate the phase plate aperture with similar results $[4,5,6]$. The effects of the "rocking" technique are clearly shown in figure 1.

With these most challenging obstacles removed, automation and system integration will be the last tasks to be solved before phase plates will become a widespread feature in biological TEM. 


\section{References:}

[1] Dubochet J. et al., Journal of Microscopy, Vol. 245 (2012), pp. 221-224.

[2] Murata K. et al., Structure, (2010), pp. 903-912.

[3] Krakow W. et al., Ultramicroscopy 2 (1976), pp. 53-67.

[4] Kawagoe Y. et al., Optics and Lasers in Engineering 3 (1982), pp. 197-218.

[5] Cotton W., Synthesis imaging in radio astronomy, A collection of Lectures from the Third NRAO Synthesis Imaging Summer School, Vol. 6 (1989), pp- 233-246.

[6] Mc Kechnie T., Applied Physics, Vol. 9 (1975), pp. 123-170.

[7] This research was supported by the German Federal Ministry of Economics and Technology (EXIST Forschungstransfer grant 03EFT4NW32).
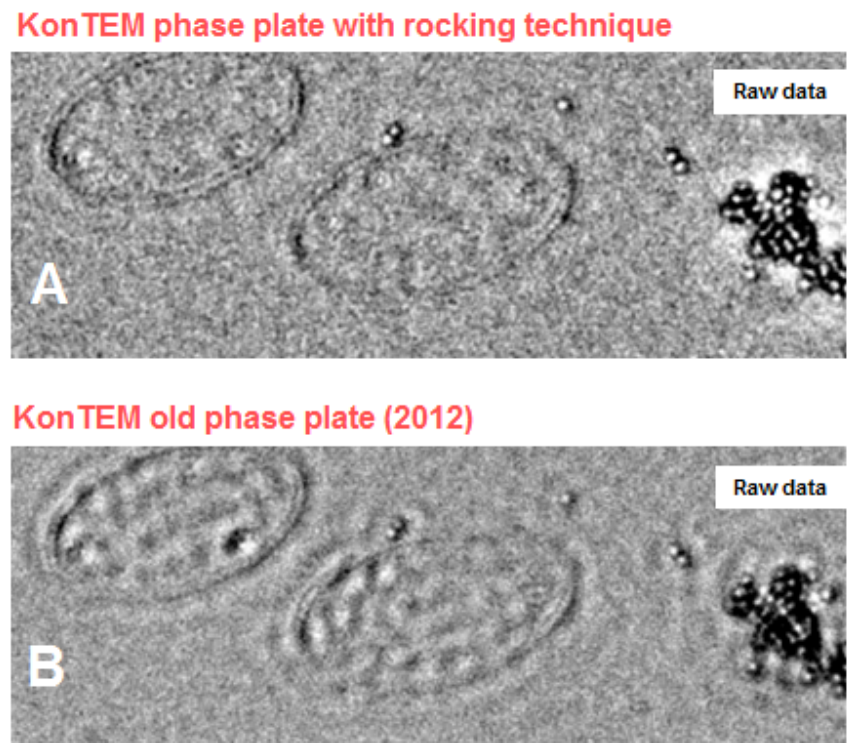

\section{No phase plate, $6 \mu \mathrm{m}$ defocus}

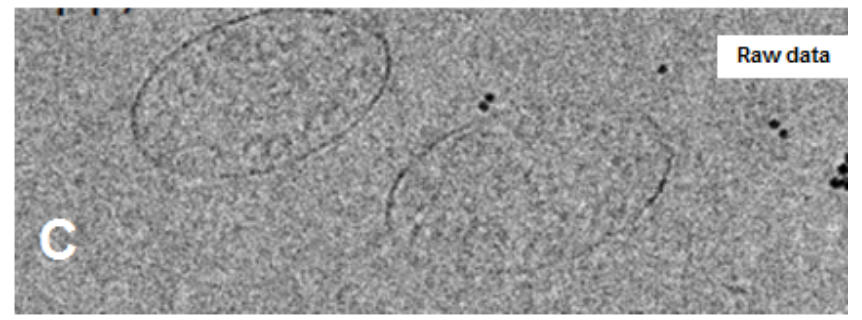

No phase plate, in focus

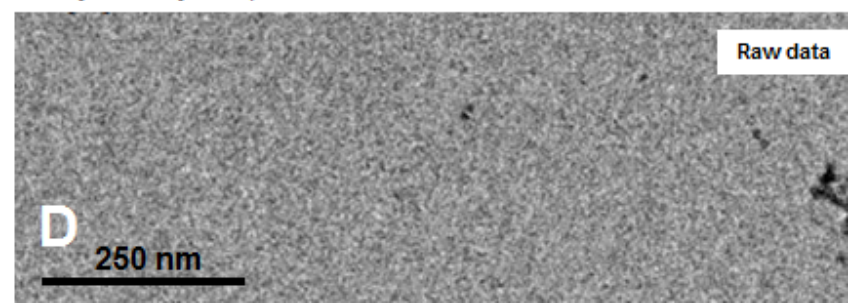

Figure 1. TEM micrographs with "rocking" phase plate (A), with standard phase plate (B), without phase plate at $6 \mu \mathrm{m}$ defocus $(\mathrm{C})$ and without phase plate in focus (D) 